\title{
Identificación de un instrumento para evaluación de la per- sonalidad, como herramienta clínica y de investigación
}

\author{
López, O ${ }^{1}$. Pinilla, L ${ }^{1}$. Manrique, I.A². Marín, S.M²
}

1 Profesor Titular, Facultad de Medicina, Fundación Universitaria Juan N. Corpas.

2 Residentes III semestre, Especialización en Terapéuticas Alternativas y Farmacología Vegetal, Fundación Universitaria Juan N. Corpas.
Correspondencia: Oscar Javier López Acero, oscar.lopez@juanncorpas.edu.co

Teléfono: 6622222, Bogotá, Colombia

Como citar: López O, Pinilla L, Manrique IA, Marín SM. Identificación de un instrumento para evaluación de la personalidad, como herramienta clínica y de investigación. Revista Cuarzo 2017; 23(1) 24-29.

Recibido: 3 de mayo de 2017 Aceptado: 10 de junio de 2017 Publicado: 30 de junio de 2017

Licencia creative commons

\section{Resumen}

INTRODUCCIÓN. Se considera que una alta proporción de enfermedades crónicas tiene un componente psicosomático y que algunos rasgos de la personalidad se relacionan con la forma en que las enfermedades se instauran y evolucionan. Para la evaluación objetiva de la personalidad han sido desarrollados instrumentos de frecuente aplicación en Psicología, pero con características que dificultan su uso como herramientas en atención médica o en investigación. El propósito del estudio fue identificar un instrumento pertinente para la evaluación de la personalidad, con el fin de ser empleado en investigación, y susceptible de ser utilizado en la clínica.

METODOLOGÍA. Se realizó un estudio descriptivo que incluyó una revisión bibliográfica y un análisis comparativo sobre cuestionarios de personalidad. Los criterios de comparación fueron: psicometría, dominios o factores, número de preguntas, modo de aplicación, tipos de personalidad, rasgos o estrategias de afrontamiento y validación.

RESULTADOS. Se encontró que no existe consenso en el concepto de personalidad, numerosos cuestionarios abordan aspectos patológicos, su aplicación requiere la intervención de psicólogos o psiquiatras, son extensos, de difícil comprensión y la mayoría carece de validación psicométrica en la población colombiana.

CONCLUSIONES. Entre los cuestionarios analizados, la Escala de Estrategias de Coping- Modificada EEC-M, orientada a establecer las estrategias de afrontamiento en función de los rasgos de personalidad, es un instrumento que se adapta a los objetivos de la presente investigación.

PALABRAS CLAVE: Personalidad, psique, enfermedad psicosomática, estrategias de afrontamiento, enfermedades crónicas. 


\section{Identification of a questionnaire for personality evaluation, as a clinical and research resource}

INTRODUCTION. It is considered that a high proportion of chronic diseases has psychosomatic component and that some personality traits are related with the way the diseases originates and evolve. For objective evaluation of the personality, instruments of frequent use in psychology have been developed, but their characteristics make difficult the application as tools in medical attention or investigation.

The purpose of the study was to identify a pertinent instrument for personality evaluation, to be used in research and susceptible to be used in clinic.

METHODOLOGY. It was made a descriptive study that included a literature review and comparative analysis about personality assessment questionnaires. The comparison criteria were: psychometrics, domains or factors, questions number, form of application, personality types, traits or facing strategies and validation.

RESULTS. It was encountered that there is no consensus about personality definition, most questionnaires perform pathologic traits, and the application requires a psychologist or a psychiatric professional, becoming so extensive and difficult in comprehension and lack of psychometric validation in Colombian population.

CONCLUSIONS. It is concluded that modified Coping Strategies Scale (EEC-M) is a questionnaire that adapts to the present research objectives.

KEYWORDS: Personality, psyche, psychosomatic illness, coping strategies, chronic diseases.

\section{Introducción}

La teoría psicosomática plantea que las formas de manifestación física de las enfermedades, se encuentran estrechamente relacionadas con las distintas maneras en que las personas canalizan las emociones y los pensamientos (1). Algunas investigaciones orientadas a identificar vínculos entre la psiquis y la enfermedad mediante el uso de imágenes diagnósticas, sugieren que situaciones traumáticas y las que generan estrés crónico activan áreas cerebrales susceptibles de desencadenar procesos patológicos (2). Así, en pacientes con cáncer se reporta morbilidad psicológica en un 50\% y depresión hasta en un 58\% de los casos, y los puntajes altos en la escala de hostilidad se asocian a un significativo incremento de la mortalidad (3).

Diversos rasgos de la personalidad se relacionan con una mayor frecuencia de enfermedades crónicas. Tal es el caso de las enfermedades cardiovasculares, y en particular la enfermedad coronaria, entre cuyos factores de riesgo ha sido identificado el Patrón de Comportamiento tipo A (PCTA), caracterizado por una elevada reactividad psicofisiológica y un aumento del tono simpático (4). Por su parte, la personalidad tipo C caracterizada por la re- presión emocional (5,) se ha relacionado de manera consistente con diversas enfermedades crónicas, entre ellas, diabetes y cáncer (6).

Especial interés representa la relación que existe entre la personalidad y las diferentes formas de afrontamiento que posee el individuo. El afrontamiento está determinado por la interacción entre el tipo de evento y los rasgos de personalidad y comprende los esfuerzos cognitivos y conductuales que se desarrollan para reducir el impacto de los estresores sobre el bienestar psicológico. Las estrategias de afrontamiento dependen de la valoración de la situación, la disponibilidad de recursos y las experiencias previas del individuo (7).

La relación entre rasgos de la personalidad, estrategias de afrontamiento y una determinada propensión a enfermar, reviste interés clínico tanto en la comprensión multidimensional de la génesis de las enfermedades como en el abordaje terapéutico de las mismas (8). Para el médico, una visión integradora del ser humano debe abarcar, además de los aspectos orgánicos, aquellos factores sociales y psicológicos susceptibles de alterar los mecanismos de respuesta biológica (9.) La exploración de la 
personalidad, o de algunos de sus componentes, es una necesidad que trasciende la práctica de los profesionales en psicología o psiquiatría y debe extenderse a la consulta del médico, especialmente en la atención de pacientes con enfermedades crónicas $(10,11)$. En este contexto es indispensable la búsqueda de instrumentos que complementen la valoración clínica y la semiología del psiquismo, con el fin de apoyar el diagnóstico sobre aspectos de la personalidad o estrategias de afrontamiento, en el ámbito de la consulta médica.

En el mismo sentido, el uso de instrumentos que hagan objetivos los rasgos de la personalidad, permitirá a las investigaciones en psicosomática establecer posibles relaciones entre personalidad y enfermedades crónicas, que conduzcan a plantear propuestas de intervención terapéutica con una visión integradora.

El propósito del estudio fue identificar un instrumento pertinente para la evaluación de la personalidad, con el fin de ser empleado en la investigación, y susceptible de ser utilizado posteriormente en la clínica.

\section{Metodología}

Se realizó un estudio descriptivo que incluyó una revisión bibliográfica y un análisis comparativo sobre cuestionarios para evaluación de la personalidad.

La población estuvo constituida por los instrumentos disponibles para la evaluación de la personalidad o de estrategias de afrontamiento.

Como instrumentos elegibles, se seleccionaron los orientados a la exploración de aspectos normales de la personalidad. Se incluyeron en el análisis aquellos que exploran tipos de personalidad, rasgos de personalidad o estrategias de afrontamiento en adultos y se excluyeron los que requieren ser administrados por un psicólogo o un psiquiatra.

Para el análisis comparativo de las escalas, se diseñó una matriz que incluyó las siguientes variables o criterios de comparación:

- Psicometría

- Dominios o factores
- Número de preguntas

- Modo de medición

- Tipos de personalidad, rasgos o estrategias de afrontamiento que evalúa

- Validación

Con los resultados preliminares, se solicitó asesoría a un grupo de expertos en pruebas psicométricas, para establecer la utilidad en la práctica clínica de los cuestionarios evaluados y se procedió a la selección de la escala más apropiada, de acuerdo con los objetivos del estudio.

\section{Resultados}

Se incluyeron en el análisis nueve cuestionarios, cuyas características se describen a continuación:

\section{EPQ-R. Cuestionario de Personalidad de Eysenck - Revisado.}

Autores: Hans J. Eynsenck y Sybil B.G. Eynseinck. Prueba psicológica de autorreporte, en la que se responde con opción dicotómica (SI/NO) a un número aproximado de 90 reactivos (según la versión de la prueba). Los aspectos que evalúa son EstabilidadNeuroticismo (N), Extraversión-Introversión (E), Normalidad-Psicoticismo (P), Labilidad o veracidad (L). La aplicación es individual o colectiva, está dirigida a mayores de 16 años y tiene una duración de 15 a 30 minutos (12). La escala se emplea con objetivos clínicos, educativos, experimentales y de selección de personal. A pesar de que algunos autores han realizado validaciones mediante análisis estructural de las dimensiones de personalidad (13) se ha sugerido que la dimensión de psicoticismo posee escasa fiabilidad y validez (14).

\section{Test de personalidad de Eysenck's (EPI) o (EPQ)}

Autores: H.J. Eysenck y S.B.G. Eysenck. Mide dos dimensiones generalizadas: extraversión-introversión y neocriticismo-estabilidad, con sus dos sub-escalas, impulsividad y socialización. Incluye una escala de sinceridad. Su aplicación puede ser individual o colectiva, en adolescentes y adultos (15). Su duración es de aproximadamente 12 minutos. A pesar del uso continuo y extendido de la prueba en diversos lugares del mundo, el único estudio de validación reportado 
la utilizó como instrumento para la validación convergente de una prueba diagnóstica para tinnitus, pero sin explorar las propiedades psicométricas de la escala (16).

\section{Inventario Multifásico de Personalidad de Min- nesota (MMPI)}

Autores: S. R. Hathway, L. C. Mchinley. Consta de tres escalas de validación y diez escalas clínicas. Es la prueba más utilizada para evaluar psicopatologías (17). En la última edición se adicionan cinco escalas: fuerza del yo, dependencia, dominancia, responsabilidad y control. Su aplicación es individual o colectiva y tiene dos versiones, una dirigida a adultos (19-65 años) y otra para adolescentes (MMPI -A) (18). En su forma original, tiene una duración de 90 a 120 minutos. Las diferentes versiones de los cuestionarios y sus traducciones han sido validadas en distintos países (19).

\section{PPG-IPG. Perfil e Inventario de Personalidad}

Autor: Leonard Gordon. La prueba es el resultado de la unión de dos instrumentos: el Perfil Personal (PPG) y el Inventario Personal (IPG). El PPG valora cuatro aspectos de la personalidad: ascendencia, responsabilidad, estabilidad emocional y sociabilidad y tiene cuatro rasgos adicionales: cautela, originalidad, relaciones personales y vigor. Su aplicación es individual o colectiva, a partir de los 14 años, y su duración es de 20 a 25 minutos. A pesar de su utilización en múltiples instancias, no se cuenta con pruebas psicométricas que sustenten su validación (20).

\section{Inventario de rasgos temperamentales (IRT)}

Autor: L.L Thurstone. Es un instrumento factorial para la evaluación de la personalidad por medio de los rasgos temperamentales, clasificados como: activo, vigoroso, impulsivo, dominante, estable, sociable y reflexivo (21). Se dirige a personas mayores de 16 años y su aplicación es individual o colectiva. La prueba tiene un tiempo de aplicación libre, con una duración aproximada de 30 minutos. A pesar de su amplio uso, no se han efectuado pruebas psicométricas para el cuestionario (22).

\section{Eneagrama}

Autor: Gurdjieff. Es una escala que evalúa los esquemas de pensamiento, el manejo emocional y la forma de actuar y clasifica la personalidad en nueve tipos fundamentales (23). La prueba se dirige a mayores de 18 años y su aplicación es individual o colectiva, con una duración de 60 minutos (24). A pesar de ser uno de los cuestionarios más antiguos para evaluación de la personalidad, su validación aún se encuentra en proceso.

\section{Escala de Estrategias de Coping- Modificada EEC-M}

Autores: Chorot y Sandín. El cuestionario valora estrategias de afrontamiento. Se considera un recurso que evalúa varios aspectos de la personalidad, por agrupar tanto los esfuerzos cognitivos como comportamentales 25. La prueba abarca 12 factores: solución de problemas, conformismo, control emocional, evitación emocional, evitación comportamental, evitación cognitiva, reacción agresiva, expresión emocional abierta, reevaluación positiva, búsqueda de apoyo social, búsqueda de apoyo, religión, refrenar afrontamiento y espera. El cuestionario comprende 69 preguntas y tiene una duración de 30 minutos 26. Cuenta con pruebas de validación en idioma español y en población colombiana (27).

\section{Cuestionario de esquemas de Young, YSQ-I2}

Desarrollado por Young en 1990, es una prueba que evalúa los esquemas maladaptativos tempranos, a los cuales clasifica en 18 grupos comprendidos dentro de cinco dominios. El cuestionario tiene aplicación individual, pero se recomienda la supervisión por un experto. Su duración es de 60 minutos. Las propiedades psicométricas y la validez de de la prueba han sido investigadas en población colombiana (28).

\section{Inventario de pensamientos automáticos}

Creado por Ruiz y Lujan en 1999, evalúa nueve aspectos: pensamiento polarizado, sobregeneralización, interpretación de pensamiento, visión catastrófica, personalización, falacia de control, falacia de justicia, razonamiento emocional y falacia de cambio. La prueba tiene una duración aproximada de 40 minutos y cuenta con numerosos estudios de validación, incluyendo población colombiana (29).

Con la información obtenida, el grupo investigador considera que entre las pruebas analizadas, la Escala de Estrategias de Coping- Modificada (EEC-M) es un instru- 
mento pertinente para los fines de la investigación, dado que permite la valoración de las formas particulares de afrontar las situaciones y establecer conductas, en concordancia con los rasgos de personalidad (30). La prueba ha sido validada en la población colombiana, es auto-administrada, sus reactivos son de fácil comprensión y tiene utilidad clínica alta, por lo que puede ser implementada en el contexto clínico.

\section{Discusión}

Para comprender la manera en que se constituye la personalidad, han sido postuladas diferentes teorías, cada una de ellas cuenta con estrategias de evaluación propias, que pretenden hacer objetivos los diversos factores que la conforman (31). El análisis comparativo realizado permitió establecer que, efectivamente, los cuestionarios existentes se basan en posturas teóricas distintas y plantean conceptos de personalidad, categorías y criterios diversos, por lo que no existe consenso acerca de los más apropiados para establecer los perfiles de personalidad. Se identifican numerosos cuestionarios con una perspectiva patológica en el abordaje de la personalidad, que no indagan sobre la conducta habitual ni la manera en que el individuo se comporta en situaciones cotidianas.

A pesar del amplio uso que tienen algunas pruebas no cuentan con estudios de psicometría que permitan establecer su validez y confiabilidad. De igual manera, carecen de validación numerosos cuestionarios en su traducción al idioma español y en su aplicación en población colombiana, aspecto de gran relevancia, considerando las variaciones socioculturales que afectan la comprensión y los resultados de las pruebas.

Entre los cuestionarios validados, se encuentra que la mayoría tiene una gran extensión que hace arduo el diligenciamiento por parte de los pacientes.

\section{Conclusiones}

A pesar de las dificultades que reviste la realización de pruebas para la evaluación objetiva de la personalidad, han sido desarrollados cuestionarios con diversos enfoques, que cuentan con estudios de psicometría y pruebas de validación. Entre ellos, la Escala de Estrategias de Coping- Modificada (EEC-M), orientada a establecer las estrategias de afrontamiento en función de los rasgos de personalidad, es un instrumento susceptible de ser empleado en la consulta médica con el fin de hacer una aproximación a la configuración psíquica de las personas. En investigación clínica, la prueba permite determinar patrones conductuales que posteriormente puedan ser relacionados con la génesis o la progresión de las enfermedades, tal como plantea la teoría psicosomática.

Se hace necesario continuar la validación de las pruebas existentes en la población colombiana y desarrollar nuevos instrumentos que permitan un mayor nivel de confianza en el propósito de evaluar los diversos componentes que configuran la personalidad.

\section{Referencias}

1. González M. T, Landero R. Síntomas psicosomáticos y teoría transaccional del estrés. Ansiedad y estrés. 2006. 12(1) $45-61$.

2. Gálvez JF. Trastornos por estrés y sus repercusiones neuropsicoendocrinológicas. rev.colomb.psiquiatr. [Internet]. 2005, 34 (1): 77-100. Disponible en: http://www. scielo.org.co/scielo.php?script=sci_arttext\&pid=S003474502005000100006\&lng=en.

3. Dresch, V. Relaciones entre personalidad y salud física-psicológica diferencias según sexo-género, situación laboral y cultura-nación. [dissertation]. Madrid: Universidad Complutense de Madrid; 2007. 337 p.

4. Moyano E, Icaza G, Mujica V, Núñez L, Leiva E, Vásquez M, Palomo I. Patrón de comportamiento tipo A, ira y enfermedades cardiovasculares (ECV) en población urbana chilena. Revista Latinoamericana de Psicología, 2011, 43(3), 443-453. Disponible en: http://www. scielo.org.co/scielo.php?script=sci_arttext\&pid=S012005342011000300005\&lng=en\&nrm=iso.

5. Anarte M T, López E A, Ramírez C, Esteve R, Evaluación del patrón de conducta tipo c en pacientes crónicos. Anales de Psicología 2000; 16. Disponible en: http://www.redalyc. org/articulo.oa?id=16716203.

6. Stefano Vinaccia. El Patrón de Conducta Tipo C en Pacientes con Enfermedades Crónicas. rev.colomb.psiquiatr. 2003, 32(2): 161-168. Disponible en: http://www. scielo.org.co/scielo.php?script=sci_arttext\&pid=S003474502003000200004\&lng=en

7. Londoño N, Henao G, Puerta I, Posada S, Arango,D, Aguirre D. Propiedades psicométricas y validación de la escala de estrategias de Coping modificada (EEC-M) en una muestra colombiana. Universitas Psychologica. 2006, 5(2): $327-350$ 
8. Benítez, M. Mecanismos de relación entre la personalidad y los procesos de salud-enfermedad. Revista de Psicología Universidad de Antioquia. 2015, 7(1): 163-184. Disponible en http://aprendeenlinea.udea.edu.co/revistas/index.php/ psicologia/article/view/25269

9. Lemos M, Restrepo DA, Richard C, Revisión crítica del concepto "psicosomático" a la luz del dualismo mente-cuerpo. Pensamiento Psicológico. 2008, 4(1) 137-147. Disponible en: http://www.redalyc.org/articulo.oa?id=80111670009.

10. Piñeros, J. Introducción a la Medicina no Tradicional. 2 ed. Bogotá: Fedicor; 1991.

11. Medicor. Cómo lograr salud mental perfecta y madurez emocional: curso básico de higiene mental. Bogotá: Fedicor; 1992.

12. Sandín B, Valiente R, Chorot P, Olmedo M, Santed M. Versión española del cuestionario EPQR-ABREVIADO (EPQR-A) (I): análisis exploratorio de la estructura factorial. Rev. Psicopatol. Psicol. Clin. [Internet]. 2002; 7(3): 195-205. Disponible en http://revistas.uned.es/index.php/ RPPC/article/view/3933

13. Forrest S, Lewis C.A, Shevlin M. Examining the factor structure and differential functioning of the Eysenck personality questionnaire revised - abbreviated. Personality and Individual Differences. 2000; 29 (3), 579-588.

14. Katz Y, Francis L. Hebrew revised Eysenck Personality Questionnaire: Short form (EPQR-S) and abbreviated form (EPQR-A). Social Behavior and Personality. 2000; 28(6), 555-560.

15. Eysenck H. Manual of the Maudsley personality inventory. London: University of London Press; 1959.

16. Zambrano R. Revisión sistemática del cuestionario de personalidad de Eysenck (Eysenck Personality Questionnarie-EPQ). Liberabit. 2011; 17(2): 147-155.

17. Vinet E, Alarcón B. "Evaluación psicométrica del inventario multifásico de personalidad de Minnesota para adolescentes (mmpi-a) en muestras chilenas. Ter. psicol . 2003; 21(2): 87-103.

18. Lucio E, Ampudia A, Duran C. Manual para la administración y calificación del MMPI-A. Versión en español. Ciudad de México: El Manual Moderno; 1998.
19. Hsu L. Diagnostic validity statistic and the MCMI-III. Psychological Assessment. 2002; 14: 10-422.

20. Cohen R, Swerdlik M. Pruebas y evaluación psicológicas: Introducción a las pruebas y a la medición. 4a . Ed. México: McGraw Hill; 2001.

21. Thurstone L. Inventario de rasgos temperamentales. IRT. México, D. F: El Manual Moderno; 2011.

22. Amigó S. La teoría del rasgo único de personalidad. Colección letras Humanas. España: Universidad Politécnica de Valencia; 2005.

23. Riso R, Hudson R. Understanding the enneagram: The practical guide to personality types. Revised edition. United States of America: Houghton Mifflin Harcourt; 2000.

24. Wagner J P and Walker R E. Reliability and validity study of a Sufi personality typology: The enneagram. J. Clin. Psychol. 1983; 39(5): 712-717.

25. Perczek R, Carver C S, Price A A., \& Pozo-Kaderman C. Coping, mood, and aspects of personality in Spanish translation and evidence of convergence with English versions. J. Pers Assess. 2000; 74(1):63-87.

26. Fernández-Abascal E G, Palmero F, Chóliz M. y Martínez F. Cuaderno de prácticas de motivación y emoción. $1^{\text {a }}$ Ed. Madrid: Pirámide; 1997.

27. Muller L, Spitz E. Multidimensional assessment of coping: validation of the- Brief COPE among French population. Encephale, 2003; 29(6): 507-518.

28. Castrillón D A, Chaves L, Ferrer A, Londoño N H, Maestre K, Marín C y Schnitter M. Validación del Young questionnaire long form-second edition (YSQ-L2) en población colombiana. Revista Latinoamericana de Psicología. 2005; 37(3): 541-560.

29. Londoño N, Álvarez C, López P y Posada S. Distorsiones cognitivas asociadas al trastorno de ansiedad generalizada. Informes psicológicos. 2005; (7): 123-126.

30. Amirkhan J H. Criterion validity of a coping measure. Journal of Personality Assessment. 1994; 62(2): 242-61.

31. Montaño M, Palacios J, Gantiva C. Teorías de la personalidad. Un análisis histórico del concepto y su medición. Psychología. Avances de la disciplina. 2009; 3 (2): 81-107. 